EPJ Web of Conferences 60, 20024 (2013)

DOI: $10.1051 /$ epjconf $/ 20136020024$

(C) Owned by the authors, published by EDP Sciences, 2013

\title{
Rare B decays with the CMS detector
}

\author{
Jacopo Pazzini ${ }^{1,2, a}$ on behalf of the CMS collaboration. \\ ${ }^{1}$ Università di Padova \\ ${ }^{2}$ INFN Padova
}

\begin{abstract}
An angular analysis of the $\mathrm{B}^{0} \rightarrow \mathrm{K}^{* 0} \mu^{+} \mu^{-}$decay is performed in $p p$ collisions at $\sqrt{s}=7 \mathrm{TeV}$, with a data sample corresponding to an integrated luminosity of $5.2 \mathrm{fb}^{-1}$ collected by the CMS experiment at the LHC. The forward-backward asymmetry of the muons $A_{F B}$, the $\mathrm{K}^{* 0}$ longitudinal polarization fraction $F_{L}$, and the differential branching fraction $\mathrm{d} \mathcal{B} / \mathrm{d} q^{2}$, are measured as function of the dimuon invariant mass squared, $q^{2}$. The results are in good agreement with the standard model expectations.
\end{abstract}

\section{Introduction}

Physics phenomena beyond the standard model (SM) of particle physics may be inferred through their influence on rare processes such as flavor-changing neutral current decays of $b$ hadrons. The semileptonic $\mathrm{B}^{0} \rightarrow \mathrm{K}^{* 0} \mu^{+} \mu^{-}$ decay (charge conjugate states are always implied unless explicitly stated) it's forbidden at tree level in the SM, resulting in small expected rates. Robust theoretical calculations are available for much of the phase space of this decay indicating that new physics could give rise to observable effects. Fig. 1 shows the Feynman diagrams of the SM decay process along with an example a supersymmetric (MSSM) $\mathrm{B}^{0} \rightarrow \mathrm{K}^{* 0} \mu^{+} \mu^{-}$decay. Two important
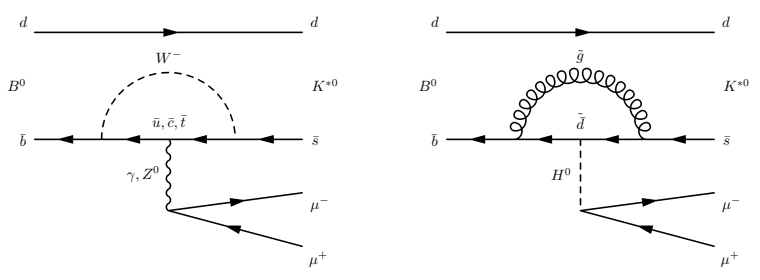

Figure 1. Examples of Feynman diagrams of the $\mathrm{B}^{0} \rightarrow \mathrm{K}^{* 0} \mu^{+} \mu^{-}$ decay within the SM (left), and within the MSSM (right).

observables related to the $\mathrm{B}^{0} \rightarrow \mathrm{K}^{* 0} \mu^{+} \mu^{-}$decay are the forward-backward asymmetry of the muons, $A_{F B}$, and the longitudinal polarization fraction of the $\mathrm{K}^{* 0}(892), F_{L}$. These can be measured as a function of the $q^{2}$ of the decay (dimuon invariant mass squared) and compared to the SM predictions [1-3]. While previous measurements by the BaBar, Belle, CDF, and LHCb experiments are consistent with the SM predictions [4-7], these measurements are still statistically limited and new results may provide an indication of new physics. The CMS experiment carried out the measurements of $A_{F B}, F_{L}$, and the differential

\footnotetext{
ae-mail: Jacopo.Pazzini@pd.infn.it
}

branching fraction $\mathrm{d} \mathcal{B} / \mathrm{d} q^{2}$ from the $\mathrm{B}^{0} \rightarrow \mathrm{K}^{* 0} \mu^{+} \mu^{-}$decay mode, using data collected from $p p$ collisions in 2011 at a center of mass energy of $7 \mathrm{TeV}$, corresponding to an integrated luminosity of $5.2 \mathrm{fb}^{-1}$ [8]. All measurements are performed in $q^{2}$ bins from 1 to $19 \mathrm{GeV}^{2}$. The $q^{2}$ bins $8.68<q^{2}<10.09 \mathrm{GeV}^{2}$ and $12.9<q^{2}<14.18 \mathrm{GeV}^{2}$, corresponding to the $\mathrm{B}^{0} \rightarrow \mathrm{K}^{* 0} \mathrm{~J} / \psi$ and $\mathrm{B}^{0} \rightarrow \mathrm{K}^{* 0} \psi^{\prime}$ decays, respectively, are used to validate the analysis and as normalization for the branching fraction measurement.

\section{Event selection}

The signal $\left(\mathrm{B}^{0} \rightarrow \mathrm{K}^{* 0} \mu^{+} \mu^{-}\right)$and normalization/control samples $\left(\mathrm{B}^{0} \rightarrow \mathrm{K}^{* 0} \mathrm{~J} / \psi\right.$ and $\left.\mathrm{B}^{0} \rightarrow \mathrm{K}^{* 0} \psi^{\prime}\right)$ were recorded with the same trigger, requiring two identified muons coming from a common vertex displaced from the primary vertex (PV) of the $p p$ collision. The offline muons selection requires two opposite charged muons, matched with the ones that caused the trigger, with pseudorapidity $|\eta(\mu)|<2.2$ and dimuon transverse momentum of $p_{\mathrm{T}}(\mu \mu)>6.9 \mathrm{GeV}$. The $\mathrm{K}^{* 0}$ candidates are reconstructed through the decay into $\mathrm{K}^{+} \pi^{-}$final state, where both the hadron track candidates must have failed the muon identification criteria and have a $p_{\mathrm{T}}>0.75 \mathrm{GeV}$. The two hadron track candidates must have an invariant mass within $80 \mathrm{MeV}$ of the nominal $\mathrm{K}^{* 0}$ mass for either the $\mathrm{K}^{+} \pi^{-}$or $\mathrm{K}^{-} \pi^{+}$hypothesis. To remove contamination from $\phi$ decays, the hadron pair invariant mass must also be greater than $1.035 \mathrm{GeV}$ when the $\mathrm{K}^{+}$mass is assigned to both hadron tracks. The $\mathrm{B}^{0}$ candidates are obtained by fitting the four charged tracks to a common vertex, and must have $\left|\eta\left(\mathrm{B}^{0}\right)\right|<2.2$ and $p_{\mathrm{T}}\left(\mathrm{B}^{0}\right)>8 \mathrm{GeV}$. The invariant mass of the four-track vertex must be within 280 $\mathrm{MeV}$ of the nominal $\mathrm{B}^{0}$ mass for either the $\mathrm{K}^{+} \pi^{-} \mu^{+} \mu^{-}$or $\mathrm{K}^{-} \pi^{+} \mu^{+} \mu^{-}$hypothesis. The four-track $\mathrm{B}^{0}$ candidate $\mathrm{CP}$ state is univocally identified depending on whether the $\mathrm{K}^{+} \pi^{-}$or the $\mathrm{K}^{-} \pi^{+}$combination is the closest to the nominal $\mathrm{K}^{* 0}$ mass. In cases where both combinations are within 
$50 \mathrm{MeV}$ from the nominal $\mathrm{K}^{* 0}$ mass no clear identification is possible, and the event is therefore rejected. Depending on the dimuon invariant mass $m_{\mu \mu}$ and its calculated uncertainty $\sigma\left(m_{\mu \mu}\right)$, the retained events are associated to the normalization or control samples if $m_{\mu \mu}$ is close to the $\mathrm{J} / \psi$ or $\psi^{\prime}$ mass, respectively. The selection requirements are optimized to maximize the $S / \sqrt{S+B}$ ratio, where $S$ is the expected signal from simulations, and $B$ is the background estimated from data sidebands. The optimization is performed on the $q^{2}$ regions far from the $\mathrm{J} / \psi$ and $\psi^{\prime}$ peaks. The reconstruction and selection efficiencies, as well as the acceptance, are obtained from Monte Carlo (MC) simulations.

\section{Analysis}

The analysis is designed to measure the forward-backward asymmetry of the muons, $A_{F B}$, the $\mathrm{K}^{* 0}$ longitudinal polarization fraction, $F_{L}$, and the differential branching fraction, $\mathrm{d} \mathcal{B} / \mathrm{d} q^{2}$, of the decay $\mathrm{B}^{0} \rightarrow \mathrm{K}^{* 0} \mu^{+} \mu^{-}$as function of the dimuon invariant mass squared. The properties of the $\mathrm{B}^{0} \rightarrow \mathrm{K}^{* 0} \mu^{+} \mu^{-}$decay can be fully described by three angles: $(i)$ the angle between the kaon momentum and the direction opposite to the $\mathrm{B}^{0}$ in the $\mathrm{K}^{* 0}$ rest frame, $\theta_{\mathrm{K}}$; (ii) the angle between the positive muon momentum and the direction opposite to the $\mathrm{B}^{0}$ in the dimuon reference frame, $\theta_{\mathrm{L}}$; (iii) the relative angle between the dimuon plane and the $\mathrm{K}^{+} \pi^{-}$plane, $\phi$, which is always integrated out in this analysis. Fig. 2 shows the definition of the relevant angles,

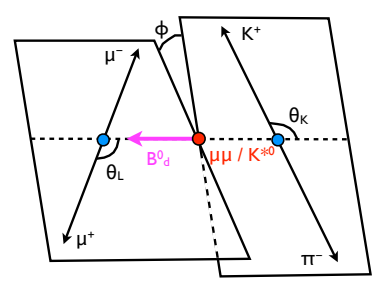

Figure 2. Representation of the $\mathrm{B}^{0} \rightarrow \mathrm{K}^{* 0} \mu^{+} \mu^{-}$decay showing the definition of the angular observables of the process.

while Eq. 1 describes the dependence of the $\mathrm{B}^{0} \rightarrow \mathrm{K}^{* 0} \mu^{+} \mu^{-}$ decay rate from the $q^{2}$ and the $\theta_{\mathrm{K}}, \theta_{\mathrm{L}}$ angular observables.

$$
\begin{aligned}
\frac{1}{\Gamma} \frac{\mathrm{d}^{3} \Gamma}{\mathrm{d} \cos \theta_{K} \mathrm{~d} \cos \theta_{L} \mathrm{~d} q^{2}} & =\frac{9}{16}\left\{\left[\frac{2}{3} F_{S}+\frac{4}{3} A_{S} \cos \theta_{K}\right]\left(1-\cos ^{2} \theta_{L}\right)\right. \\
& +\left(1-F_{S}\right)\left[2 F_{L} \cos ^{2} \theta_{K}\left(1-\cos ^{2} \theta_{L}\right)\right. \\
& +\frac{1}{2}\left(1-F_{L}\right)\left(1-\cos ^{2} \theta_{K}\right)\left(1+\cos ^{2} \theta_{L}\right) \\
& \left.\left.+\frac{4}{3} A_{F B}\left(1-\cos ^{2} \theta_{K}\right) \cos \theta_{L}\right]\right\}
\end{aligned}
$$

Although the $\mathrm{K}^{+} \pi^{-}$invariant mass must be consistent with a $\mathrm{K}^{* 0}$, there can be contributions from a spinless (S-wave) $\mathrm{K}^{+} \pi^{-}$combination. The $\mathrm{S}$-wave fraction is described in Eq. 1 by the term $F_{S}$, while the interference between the $\mathrm{S}$-wave and P-wave decays is described by $A_{S}$. The results of this analysis are extracted for each $q^{2}$ bin from unbinned maximum likelihood fits to three variables: $\cos \theta_{\mathrm{K}}$, $\cos \theta_{\mathrm{L}}$, and the $\mathrm{K}^{+} \pi^{-} \mu^{+} \mu^{-}$invariant mass. The values of
$F_{S}$ and $A_{S}$ are extracted from the fit to the normalization $\mathrm{B}^{0} \rightarrow \mathrm{K}^{* 0} \mathrm{~J} / \psi$ sample, and used in the fit to the signal sample for each $q^{2}$ bin with Gaussian constraints defined by the uncertainty resulting from the $\mathrm{B}^{0} \rightarrow \mathrm{K}^{* 0} \mathrm{~J} / \psi$ fit. The differential branching fraction $\mathrm{d} \mathcal{B} / \mathrm{d} q^{2}$ is measured relative to the normalization sample $\mathrm{B}^{0} \rightarrow \mathrm{K}^{* 0} \mathrm{~J} / \psi$ according to Eq. 2.

$$
\frac{\mathrm{d} \mathcal{B}\left(\mathrm{B}^{0} \rightarrow \mathrm{K}^{* 0} \mu^{+} \mu^{-}\right)}{\mathrm{d} q^{2}}=\frac{Y_{S}}{Y_{N}} \frac{\epsilon_{N}}{\epsilon_{S}} \frac{\mathcal{B}\left(\mathrm{B}^{0} \rightarrow \mathrm{K}^{* 0} \mathrm{~J} / \psi\right)}{\mathrm{d} q^{2}}
$$

Where $Y_{S}\left(Y_{N}\right)$ and $\epsilon_{S}\left(\epsilon_{N}\right)$ are the yield and the total efficiency of the signal (normalization) channel respectively, and $\mathcal{B}\left(\mathrm{B}^{0} \rightarrow \mathrm{K}^{* 0} \mathrm{~J} / \psi\right)$ is the world average branching fraction for the normalization channel [9]. The yields are obtained by the fit to the $\mathrm{K}^{+} \pi^{-} \mu^{+} \mu^{-}$invariant mass distributions, while the the efficiencies are obtained by integrating over the angular variables using the results from the previous fits. There are two contributions for the background: the peaking background, due to the remaining $\mathrm{B}^{0} \rightarrow \mathrm{K}^{* 0} \mathrm{~J} / \psi$ and $\mathrm{B}^{0} \rightarrow \mathrm{K}^{* 0} \psi^{\prime}$ events not removed by the dimuon invariant mass cuts, and the combinatorial background, due to the unavoidable fraction of mis-reconstructed $\mathrm{B}^{0}$ from randomly associated muons to hadrons. In the peaking background events the dimuon mass is reconstructed far from the true $\mathrm{J} / \psi\left(\psi^{\prime}\right)$ mass, resulting in a $\mathrm{K}^{+} \pi^{-} \mu^{+} \mu^{-}$invariant mass shifted from the true $\mathrm{B}^{0}$ mass. The shape of this background is obtained from simulation, while the yield obtained in the simulation is normalized comparing the reconstructed $\mathrm{B}^{0} \rightarrow \mathrm{K}^{* 0} \mathrm{~J} / \psi$ $\left(\mathrm{B}^{0} \rightarrow \mathrm{K}^{* 0} \psi^{\prime}\right)$ yields in data and simulation.

\section{Results}

The first fit to data is performed on the normalization sample $\mathrm{B}^{0} \rightarrow \mathrm{K}^{* 0} \mathrm{~J} / \psi$, obtaining a yield of 47000 signal events, as shown in Fig. 3. The results are used

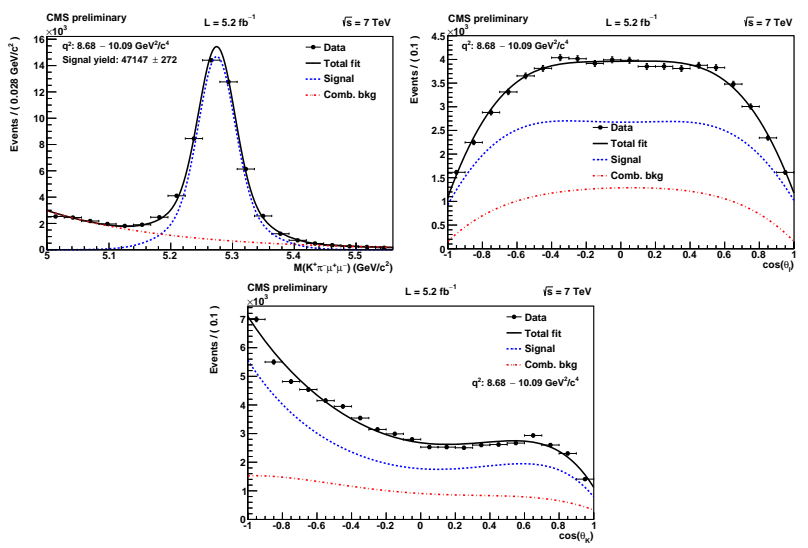

Figure 3. The $\mathrm{K}^{+} \pi^{-} \mu^{+} \mu^{-}$invariant mass (top left), $\cos \theta_{\mathrm{L}}$ (top right), and $\cos \theta_{\mathrm{K}}$ (bottom) distributions for the $q^{2}$ bin associated to the $\mathrm{B}^{0} \rightarrow \mathrm{K}^{* 0} \mathrm{~J} / \psi$, along with the projections of the fit.

to validate the fit and to estimate the $F_{S}$ and $A_{S}$ contributions to be used in the signal $q^{2}$ bins. The Swave fraction is measured to be $F_{S}=0.01 \pm 0.01$ (stat.), 
and the scalar-vector interference term is determined to be $A_{S}=-0.10 \pm 0.01$ (stat.). Along with $F_{S}$ and $A_{S}$, it is measured the longitudinal $\mathrm{K}^{* 0}(892)$ polarization fraction $F_{L}=0.554 \pm 0.004$ (stat.), and the dimuon forwardbackward asymmetry $A_{F B}=-0.004 \pm 0.004$ (stat.) in the normalization sample. The $F_{L}$ and $A_{F B}$ results are found to be compatible with the expected world average values, and consistent with the hypothesis of no asymmetry [10]. The fits are also performed in the $\mathrm{B}^{0} \rightarrow \mathrm{K}^{* 0} \psi^{\prime}$ $q^{2}$ region, where 3200 signal events yielded results of $F_{L}=0.509 \pm 0.016$ (stat.), which is consistent with the world average value, and $A_{F B}=0.013 \pm 0.014$ (stat.), compatible with no asymmetry, as expected [10]. Six signal $q^{2}$ regions are studied in the range from 1 to $19 \mathrm{GeV}^{2}$, with the exclusion of the $\mathrm{B}^{0} \rightarrow \mathrm{K}^{* 0} \mathrm{~J} / \psi$ and $\mathrm{B}^{0} \rightarrow \mathrm{K}^{* 0} \psi^{\prime}$ bins. Clear signals are seen in each bin, with yields from $23 \pm 6$ to $103 \pm 12$ events. From the fit, the $\mathrm{K}^{* 0}$ (892) longitudinal polarization fraction, $F_{L}$, and the muon forwardbackward asymmetry, $A_{F B}$, are obtained for each $q^{2}$ bin. Fig. 4 shows the comparison between these results and the SM predictions [10]. Using the yields for the signal and
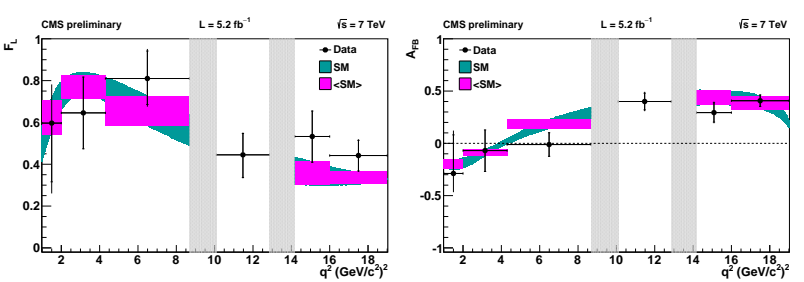

Figure 4. Results of the measurements of $F_{L}$ (left) and $A_{F B}$ (right) in $q^{2}$ bins. The statistical (total) errors on the data points are represented by the shorter (longer) error bars. The gray regions correspond to the $\mathrm{J} / \psi$ and $\psi^{\prime}$ bins. The SM prediction is given by the cyan band. The average SM prediction for each $q^{2}$ bin is shown by the magenta boxes.

the normalization modes obtained from the previous fits and the world average branching fraction of the normalization mode [9], the branching fraction for $\mathrm{B}^{0} \rightarrow \mathrm{K}^{* 0} \mu^{+} \mu^{-}$ is obtained as a function of $q^{2}$. The result is shown in Fig. 5 along with the SM expectations [10]. The angular observ-

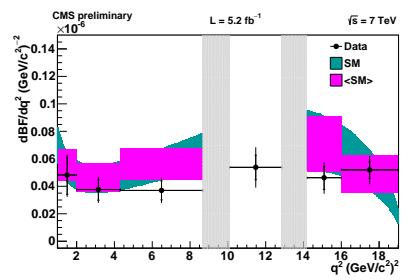

Figure 5. Results of the measurements of $\mathrm{d} \mathcal{B} / \mathrm{d} q^{2}$ in $q^{2}$ bins. The statistical (total) errors on the data points are represented by the shorter (longer) error bars. The gray regions correspond to the $\mathrm{J} / \psi$ and $\psi^{\prime}$ bins. The SM prediction is given by the cyan band. The average SM prediction for each $q^{2}$ bin is shown by the magenta boxes.

ables can be theoretically predicted with good control of the relevant form factors in the low dimuon invariant mass region. It is therefore performed the measurements of $F_{L}$,
$A_{F B}$ and $\mathrm{d} \mathcal{B} / \mathrm{d} q^{2}$ in the $1<q^{2}<6 \mathrm{GeV}^{2}$ region. Fig. 6 shows the fit to data observables in the considered $q^{2}$ region. The resulting values are: $F_{L}=0.68 \pm 0.10$ (stat.) \pm 0.02 (syst.), $A_{F B}=-0.07 \pm 0.12$ (stat.) \pm 0.01 (syst.), and $\mathrm{d} \mathcal{B} / \mathrm{d} q^{2}=(4.4 \pm 0.6$ (stat.) \pm 0.7 (syst. $\left.)\right) \times 10^{-8} \mathrm{GeV}^{-2}$ (where the systematic uncertainty on $\mathrm{d} \mathcal{B} / \mathrm{d} q^{2}$ does not include the $4.6 \%$ normalization uncertainty). These results are consistent with the $\mathrm{SM}$ predictions of $F_{L}=0.74_{-0.07}^{+0.06}, \quad A_{F B}=-0.04 \pm 0.03$, and $\mathrm{d} \mathcal{B} / \mathrm{d} q^{2}=$ $\left(4.9_{-1.1}^{+1.0}\right) \times 10^{-8} \mathrm{GeV}^{-2}[11]$.

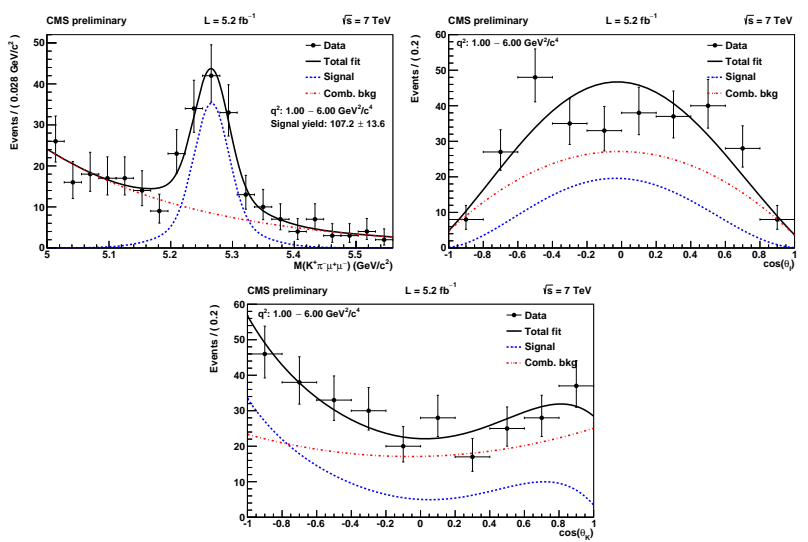

Figure 6. The $\mathrm{K}^{+} \pi^{-} \mu^{+} \mu^{-}$invariant mass (top left), $\cos \theta_{\mathrm{L}}$ (top right), and $\cos \theta_{\mathrm{K}}$ (bottom) distributions for the $1<q^{2}<6 \mathrm{GeV}^{2}$ region, along with the projections of the fit.

\section{Summary}

The CMS experiment carried out an angular analysis of the $\mathrm{B}^{0} \rightarrow \mathrm{K}^{* 0} \mu^{+} \mu^{-}$decay using data recorded during 2011 corresponding to an integrated luminosity of $5.2 \mathrm{fb}^{-1}$. Unbinned maximum likelihood fits were performed in bins of dimuon invariant mass squared $\left(q^{2}\right)$ to obtain the values of the $\mathrm{K}^{* 0}$ (892) longitudinal polarization fraction, $F_{L}$, and the forward-backward muon asymmetry, $A_{F B}$. Using these results, unbinned maximum likelihood fits to the $\mathrm{K}^{+} \pi^{-} \mu^{+} \mu^{-}$invariant mass in $q^{2}$ bins are used to extract the differential branching fraction $\mathrm{d} \mathcal{B} / \mathrm{d} q^{2}$. No deviations from the standard model predictions are found.

\section{References}

[1] F. Krüger et al., Phys. Rev. D61 114028 (2010)

[2] C. Bobeth et al., JHEP07 098 (2010)

[3] C. Bobeth et al., JHEP01 107 (2012)

[4] BaBar Collab., Phys. Rev. D79 031102 (2009)

[5] Belle Collab., Phys. Rev. Lett.103 171801 (2009)

[6] CDF Collab., Phys. Rev. Lett.108 081807 (2012)

[7] LHCb Collab., Phys. Rev. Lett.108 181806 (2012)

[8] CMS Collab., CMS-PAS-BPH-11-009

[9] J. Beringer et al. (Particle Data Group), Phys. Rev. D86 010001 (2012)

[10] C. Bobeth et al., Phys. Rev. D87 034016 (2012)

[11] C. Bobeth et al., JHEP07 067 (2011) 